\title{
Linkage mapping in tetraploid willows: segregation of molecular markers and estimation of linkage phases support an allotetraploid structure for Salix alba $\times$ Salix fragilis interspecific hybrids
}

\author{
G Barcaccia ${ }^{1}$, S Meneghetti ${ }^{1}$, E Albertini ${ }^{2}$, L Triest $^{3}$ and M Lucchin ${ }^{1}$ \\ ${ }^{1}$ Department of Environmental Agronomy and Crop Production, University of Padova, Agripolis, Via Romea 16, I-35020 Legnaro, \\ Padova, Italy; ${ }^{2}$ Department of Plant Biology and Agro-environmental Biotechnology, University of Perugia, Borgo XX Giugno 74, \\ I-06121 Perugia, Italy; ${ }^{3}$ Plant Science and Nature Management, Vrije Universiteit Brussel, Pleinlaan 2, B-1050 Brussels, Belgium
}

\begin{abstract}
Salix alba-Salix fragilis complex includes closely related dioecious polyploid species, which are obligate outcrossers. Natural populations of these willows and their hybrids are represented by a mixture of highly heterozygous genotypes sharing a common gene pool. Since nothing is known about their genomic constitution, tetraploidy $(2 n=4 x=76)$ in willow species makes basic and applied genetic studies difficult. We have used a two-way pseudotestcross strategy and singledose markers (SDMs) to construct the first linkage maps for both pistillate and staminate willows. A total of 242 amplified fragment length polymorphisms (AFLPs) and 50 selective amplifications of microsatellite polymorphic loci (SAMPL) markers, which showed 1:1 segregation in the $F_{1}$ mapping populations, were used in linkage analysis. In S. alba, 73 maternal and 48 paternal SDMs were mapped to 19 and 16 linkage groups covering 708 and $339 \mathrm{cM}$, respectively. In S. fragilis, 13 maternal and 33 paternal SDMs were mapped
\end{abstract}

in six and 14 linkage groups covering 98 and $321 \mathrm{cM}$, respectively. For most cosegregation groups, a comparable number of markers linked in coupling and repulsion was identified. This finding suggests that most of chromosomes pair preferentially as occurs in allotetraploid species exhibiting disomic inheritance. The detection of 10 pairs of marker alleles from single parents showing codominant inheritance strengthens this hypothesis. The fact that, of the 1122 marker loci identified in the two male and female parents, the vast majority $(77.5 \%)$ were polymorphic and as few as $22.5 \%$ were shared between parental species highlight that $S$. alba and $S$. fragilis genotypes are differentiated. The highly difference between $S$. alba- and $S$. fragilis-specific markers found in both parental combinations (on average, 65.3 vs $34.7 \%$, respectively) supports the (phylogenetic) hypothesis that $S$. fragilis is derived from $S$. alba-like progenitors. Heredity (2003) 90, 169-180. doi:10.1038/sj.hdy.6800213

Keywords: polyploids; willow; SDMs; AFLPs; SAMPLs; linkage maps

\section{Introduction}

Salix is a large heterogeneous genus, comprising over 300 species that show considerable variation in plant size, growth habit and crown architecture, ranging from small dwarf shrubs to large trees. The true willows, Salix alba L. (white willow) and Salix fragilis L. (crack willow), are typical arborescent pioneer species in alluvial plains and riparian zones; these species belong to the subgenus Amerina Dum., section Fragiles Fr. Salix fragilis is mainly tetraploid $(2 n=4 x=76)$, but diploid $(2 n=2 x=38)$ and hexaploid $(2 n=6 x=114)$ types are also possible, whilst $S$. alba is only tetraploid and includes four distinct subspecies; ssp. alba, ssp. coerulea Sm., ssp. vitellina (L.) (Arcang.) and ssp. micans Anderss. (Paiero and Martini, 1988). Salix $\times$ rubens Schrank is a species that arose through natural hybridisation between $S$. alba and $S$. fragilis, and includes a wide range of variants from typical S. alba to $S$. fragilis-like individuals (Newsholme,

Correspondence: G Barcaccia, Department of Environmental Agronomy and Crop Production, University of Padova, Agripolis, Via Romea 16, I-35020 Legnaro, Padova, Italy; E-mail: gianni.barcaccia@unipd.it Received 7 January 2002; accepted 26 September 2002
1992). Salix babylonica L., S. pentandra L. and S. triandra L. are the only species that successfully intercross with both S. alba and S. fragilis (Neumann, 1981). Although they have similar ecological characteristics, S. fragilis and $S$. alba are distinguishable in terms of their temperature requirements and areas of spontaneous occurrence; the former is native to Western Asia and naturalised in central and Northern Europe, and the latter more wide spread in temperate regions and the Mediterranean basin (Skvortsov, 1973).

Morphology indicates that $S$. alba, S. fragilis, $S . \times$ rubens and their natural hybrids form a polyploid complex of closely related willows. This complex includes mostly tetraploid dioecious trees, which are obligate outcrossers. As a consequence, natural populations of these willows can be represented by a mixture of highly heterozygous genotypes sharing a common gene pool as a result of recurring hybridisation. Given the breeding system of willow species, genetic variation within populations is usually as high as the genetic differentiation between populations, whereas gene flow between species is fairly moderate (Barcaccia et al, 2000a). Natural stands and unimproved populations of willows belonging to this complex are widespread throughout Europe, where habitat boundaries frequently overlap and mixed communities can 
also exist. Based on morphology, both balanced S. alba-S. fragilis hybrids and introgressive hybrids, deriving from repeated backcrossing of hybrids to parental types, seem to dominate in the field (De Bondt, 1996; Triest et al, 2000). The analysis of leaves, buds and twigs have indicated that many potential hybrids are present within natural populations (Triest, 2001). This finding agrees with the observations of several botanists (Rechinger, 1964; Skvortsov, 1973; De Langhe et al, 1983; Meikle, 1992) that the vast majority of willows in the $S$. alba-S. fragilis complex could be hybrids or introgressants. However, owing to their continuous variation, most phenotypic features have low diagnostic value for identifying interspecific hybrid constitutions, assessing introgression patterns, and defining genetic variation structure and relatedness at the population level. Recent molecular studies carried out in controlled crosses and field clones contradict traditional hypothesis on the extensive occurrence of hybrids in the $S$. alba-S. fragilis complex. In particular, molecular markers revealed that both species have kept their gene pools well separated and that interspecific hybridisation actually does not seem to be a dominating process (Triest et al, 2000). Consequently, the taxonomic classification of pure species as well as the identification of hybrid individuals remains a matter of debate.

New biotechnological developments have expanded the range of plant DNA polymorphism assays for characterising and investigating germplasm resources and genetic relatedness, as well as for linkage mapping, gene targeting and assisted breeding (Powell et al, 1996). These techniques include not only RFLP markers, but also PCR-based molecular markers. The latter type of markers offers an almost unlimited supply of molecular traits for distinctive fingerprinting of plant materials without prior knowledge of target DNA sequences. The area of willow research showing the greatest development with respect to the use of molecular marker technology is that of population genetics (Beismann et al, 1997; Triest et al, 1999, 2000; Triest, 2001), whereas information on basic genomics is scanty (Thibault, 1997; Triest et al, 1997; Alstrom-Rapaport et al, 1998).

PCR-based markers have proved very useful for developing and integrating genetic maps of many plant species. In particular, the AFLP (amplified fragment length polymorphism) technology (Zabeau and Vos, 1992) has been combined with the advantageous features of single sequence repeats (SSRs) into a single assay, which allows the selective amplification of microsatellite polymorphic loci (SAMPL) (Morgante and Vogel, 1994). The key to success is because of their multilocus nature, which allows many chromosome traits to be checked simultaneously. However, the extreme complexity of the banding profiles visualised present difficulties in identifying marker alleles, and, in consequence, performing genetic analyses. Moreover, in some polyploid species, determining the inheritance patterns is a problematic task owing to the uncertainty of the genome constitution.

Tetraploidy in $S$. alba and $S$. fragilis $(2 n=4 x=76)$ makes genetic analysis far from easy. Nothing is known about willow genomic constitution and whether species are autopolyploid or allopolyploid. Moreover, cytological observations of the pairing behaviour of tetraploid willow, to reveal bivalent or multivalent formation, are difficult owing to their high chromosome number and small chromosome size. Since willows have a high basic chromosome number $(x=19)$, species with $2 n=38$ may be ancient polyploid derivatives (Stebbins, 1950; Lewis, 1980) that behave as functionally diploids (Aravanopoulos et al, 1993; Triest et al, 1998). A practical way to handle molecular marker data in polyploids is to treat each band as a marker allele of a single locus of a given genotype and to infer its allelic dose by studying the segregation ratio among progeny (Wu et al, 1992; Hackett et al, 1998; Ripol et al, 1999; Porceddu et al, 2002). A band present in a single dose (single-dose marker, SDM) in a tetraploid plant, for example, will be inherited by half of the gametes. If such a plant is crossed with a plant lacking that marker, the segregation ratio expected in the progeny would be 1:1 (presence vs absence). Whereas when selfed or crossed with a plant sharing that marker, the progeny would be expected to have a segregation ratio of 3:1 (presence $v$ s absence), irrespective of disomic or tetrasomic inheritance. The situation becomes more complicated when the segregation ratio of two SDMs has to be deciphered. Two SDMs may be either linked in coupling on the same chromosome, linked in repulsion on two homologous chromosome or completely unlinked. Both the expected segregation ratios and linkage equations for SDMs linked in coupling are equivalent to those for diploids. The same holds for repulsion phase linkages in species with a preferential pairing, such as allopolyploids (Wu et al, 1992). The definition of the expected segregation ratios is more complicated in autopolyploids and relies on many factors, including the ploidy level, the chromosome pairing behaviour and the extent of double reduction (Wu et al, 1992).

The genetic analysis in willow has been restricted not only because of genome complexity, but also because of the lack of suitable marker systems and the need of experimental populations amenable to genetic analysis. As a result, neither genetic maps have been defined nor segregation patterns or recombination estimates have been reported for tetraploid willow. Molecular investigations of diploid species belonging to the $S$. alba-S. fragilis complex could provide valuable data about the genetic structure of the willow genome and it is likely that some of the information would also be applicable to tetraploid willow. However, it cannot be assumed that the map distances calculated for diploid willow will be the same in the tetraploid genome and it may not be possible to map all of the characteristics observed in tetraploid willow (particularly quantitative traits) in a diploid background. The development of a map for the tetraploid genome using interspecific $S$. alba-S. fragilis hybrids and reciprocals would also be very useful for estimating the genomic affinity and understanding the recombination potential between the two species.

This paper deals with the construction of the first linkage maps for male and female willows based on a two-way pseudotestcross strategy. Only single-dose AFLP and SAMPL markers polymorphic between parents that showed 1:1 segregation in the $F_{1}$ mapping populations were used in linkage analysis.

\section{Materials and methods}

\section{Plant material and controlled matings}

Two segregating $F_{1}$ populations of 69 plants each were produced by performing reciprocal crosses between 
S. alba and S. fragilis. Both the male and female $S$. fragilis clonal genotypes originated from Brno (Czech Republic), whereas the female and male parents of $S$. alba belong to natural populations from Scarperia (Florence, Italy) and Panfilia (Ferrara, Italy), respectively. Seeds were germinated in jiffy pots and plantlets were grown in the greenhouse.

DNA isolation and AFLP and SAMPL marker analysis Genomic DNA of single $F_{1}$ plants and parents was extracted using the CTAB procedure (Doyle and Doyle, 1990) and then restricted and ligated to adapters according to Barcaccia et al (1999). Briefly, genomic DNA (500 ng) was digested and ligated for $4 \mathrm{~h}$ at $37^{\circ} \mathrm{C}$ using EcoRI (or PstI) and MseI enzymes (5 U each), $1 \mathrm{U}$ of T4 ligase, $50 \mathrm{pmol}$ of MseI-adaptor, $5 \mathrm{pmol}$ of EcoRIadaptor in $1 \times \mathrm{RL}$ buffer $(20 \mathrm{mM}$ Tris-acetate, $20 \mathrm{mM}$ magnesium acetate, $100 \mathrm{mM}$ potassium acetate, $5 \mathrm{mM}$ DTT, $2.5 \mathrm{mg}$ BSA) added with ATP to a final concentration of $10 \mathrm{mM}$. Then, $5 \mu \mathrm{l}$ of eight-fold diluted digested and ligated DNA was preamplified to select, and bulk restriction fragments to the correct size and configuration in $20 \mu \mathrm{l}$ reaction mixture containing $75 \mathrm{ng}$ of EcoRI+N (or Pst $\mathrm{I}+\mathrm{N})$ and $\mathrm{Mse} \mathrm{I}+\mathrm{N}$ primers, $1 \times \mathrm{PCR}$ buffer $(50 \mathrm{mM}$ $\mathrm{MgCl}, 1.5 \mathrm{mM} \mathrm{MgCl} 2,10 \mathrm{mM}$ Tris- $\mathrm{HCl}), 10 \mathrm{mM}$ dNTPs and $1 \mathrm{U}$ of Taq DNA polymerase. The cycling conditions were 1 cycle of $45 \mathrm{~s}$ at $94^{\circ} \mathrm{C}, 30 \mathrm{~s}$ at $65^{\circ} \mathrm{C}, 1 \mathrm{~min}$ at $72^{\circ} \mathrm{C}$ and a touch-down profile $\left(13\right.$ cycles with $-0.7^{\circ} \mathrm{C} /$ cycle) for the annealing step followed by 18 cycles at $55.9^{\circ} \mathrm{C}$ annealing temperature and finally by an extension cycle of $5 \mathrm{~min}$ at $72^{\circ} \mathrm{C}$.

AFLP marker analysis was performed with a $\left[{ }^{33} \mathrm{P}\right]-$ labelled EcoRI+3 (or PstI+2) primer and an unlabelled MseI+3 primer. Sequences of restriction site-related primers were as follows: EcoRI-primer: AGACTGCGTACCAATTC; PstI-primer: GACTGCGTACATGCAG; and MseI-primer: GACGATGAGTCCTGAGTAA. Each $20 \mu \mathrm{l}$ PCR reaction contained $0.5 \mu \mathrm{l}$ of the preamplified DNA, $50 \mathrm{ng}$ of labelled EcoRI- or PstI-primer, $30 \mathrm{ng}$ of unlabelled MseI-primer, $2 \mu \mathrm{l}$ of PCR buffer, $4 \mathrm{mM}$ dNTPs and $0.4 \mathrm{U}$ of Taq DNA polymerase (Barcaccia et al, 1998).

SAMPL marker analysis was performed using a procedure identical to that adopted for AFLP reported above, except for one of the primers used in the second amplification. Instead, the hot-PCR was performed with the [ $\left.{ }^{33} \mathrm{P}\right]$-labelled $A s 1-$ or As2-primer and a standard MseI-primer with three selective nucleotides (De Simone et al, 1997). Sequences of adjacent-microsatellite primers are as follows: As1-primer: CACACACACACACACTATAT; and As2-primer: GAGAGAGAGAGAGAGATA.

AFLP and SAMPL primer combinations (ie selective bases) are in Table 1.

Both AFLP and SAMPL amplifications were carried out using the same amplification conditions described above. After PCR, $8 \mu \mathrm{l}$ of loading buffer $(98 \%$ formamide, $10 \mathrm{mM}$ EDTA, $0.005 \%$ each of xylene-cyanol and bromophenol-blue) was added to each tube. Samples were denatured at $90^{\circ} \mathrm{C}$ for $5 \mathrm{~min}$ and then immediately placed on ice. For each sample, $5 \mu \mathrm{l}$ were loaded onto a $6 \%$ polyacrylamide gel $(60 \mathrm{~cm} \times 30 \mathrm{~cm} \times 0.4 \mathrm{~mm})$, which was run for $2 \mathrm{hr}$ and $45 \mathrm{~min}$ at $80 \mathrm{~W}$. Markers were visualised on autoradiograms after $18 \mathrm{hr}$ exposure at $-80^{\circ} \mathrm{C}$ with intensifying screens.

\section{Segregation analysis}

A molecular marker segregating from one parent may be present as a single dose (SDM), double dose (DDM), triple dose (TDM) alleles, etc. Whereas SDMs are expected to segregate in the same way regardless of the ploidy level and genome constitution, the expected segregation ratio of higher dose markers, such as DDMs and TDMs, varies according to the genome constitution (autopolyploidy $v s$ allopolyploidy), the ploidy level, and the pairing behaviour (bivalents, trivalents, quadrivalents). A DDM of an allotetraploid, for example, may be inherited in all or three out of four of the gametes depending on whether the two copies of the marker allele belong to homologous or homeologous chromosomes. The expected segregation ratio presence vs absence in gametes of autopolyploids with bivalent pairing at meiosis is, instead, $(3 h-2):(h-2)$ where $h$ represents the ploidy level (Sorrells, 1992). Therefore, a DDM of an autotetraploid can be inherited in five out of the six possible gametes.

In species with a tetraploid genome, such as willow, it is therefore very important to distinguish SDMs from non-SDMs. The segregation patterns (presence vs absence) of marker alleles observed in the $F_{1}$ mapping

Table 1 AFLP (Eco/Mse and Pst/Mse) and SAMPL (As/Mse) primer combinations assayed in Salix alba-S. fragilis

\begin{tabular}{|c|c|c|c|c|c|c|c|c|}
\hline & $\mathrm{ECO}+\mathrm{CAC}$ & $E c o+C A$ & $\mathrm{Eco}+\mathrm{CC}$ & $P_{s t}+A A$ & $P s t+A G$ & $P s t+A T$ & As1 & As2 \\
\hline $\mathrm{Mse}+\mathrm{CCA}$ & & & & 1,2 & 1 & 1,2 & & 3 \\
\hline Mse+CAA & 3 & & & & 1,2 & 1 & & \\
\hline Mse+CAC & 3 & & & & 1,2 & 1 & & \\
\hline $\mathrm{Mse}+\mathrm{CAG}$ & & & & & 1 & 1,2 & 3 & 3 \\
\hline Mse+CAT & & & & & 1 & 1 & & \\
\hline Mse+AGG & & 1,2 & & & & & 3 & \\
\hline Mse+AAG & & & 1,2 & & & & & \\
\hline Mse+AGT & & & 1,2 & & & & & \\
\hline Mse+ATC & 3 & & 1,2 & & & & 3 & \\
\hline $\mathrm{Mse}+\mathrm{AA}$ & & & & & & & 3 & 1,2 \\
\hline $\mathrm{Mse}+\mathrm{AC}$ & & & & & & & 3 & 1,2 \\
\hline$M s e+\mathrm{AT}$ & 3 & & & & & & & \\
\hline
\end{tabular}

$1=$ tested in parental plants and used to analyse the $S$. alba $\times S$. fragilis $\mathrm{F}_{1}$ progenies; $2=$ tested in parental plants and used to analyse the S. fragilis $\times S$. alba $\mathrm{F}_{1}$ progenies; 3 =tested in parental plants only (not used for the analysis of $\mathrm{F}_{1}$ progenies because of the low multiplex ratio and the poor fingerprint quality). 
populations were assayed by $\chi^{2}$ analysis to test the goodness of fit with the 1:1 and 3:1 ratios expected, respectively, for marker loci that were either polymorphic or shared between parents. In the case of autotetraploidy, parental marker loci genotypes would be simplex $(a---)$ by nulliplex (- - -) and simplex (a- - -) by simplex $(a--)$, whereas in case of allotetraploidy they would be heterozygous ( $a-)$ by homozygous (- -$)$ and heterozygous $(a-)$ by heterozygous $(a-)$.

The observed numbers of AFLP and SAMPL species-specific polymorphisms, segregating parental (both male and female) SDMs and mapped marker loci were analysed by $2 \times 2$ contingency tests in order to compare the genomic affinity between the two species and the efficiency of the two molecular marker systems.

\section{Linkage mapping}

The $F_{1}$ progenies were scored for marker loci polymorphisms. All AFLP and SAMPL marker alleles polymorphic between parents that fitted a 1:1 segregation ratio were assayed by the Mather's $\chi^{2}$ test to assess association (Mather, 1957). Salix alba- and S. fragilisspecific SDMs were ordered in maternal and paternal cosegregation groups. SDMs of each cosegregation group were then tested for the linkage phase on the basis of parental vs recombinant patterns. The number of SDMs linked in coupling and the number of those linked in repulsion calculated over all pairwise comparisons were assayed to fit the 1:1 proportional ratio. When the difference between coupling and repulsion phase marker combinations was not statistically significant, chromosome pairing was considered preferential. However, if the difference was significant, chromosome pairing was considered to be random (Wu et al, 1992).

Marker segregation data were then analysed with JoinMap 3.0 (van Oijen and Voorrips, 2001) by treating segregation data of markers as a 'backcross' $\left(\mathrm{BC}_{1}\right)$ population, that is, by following a two-way pseudotestcross mapping strategy (Grattapaglia and Sederoff, 1994). Data were also analysed by using the 'crosspollination' (CP) population type option, that is, an $F_{1}$ population resulting from a cross between two heterogeneous parents, which were, respectively, simplex and nulliplex at the loci being tested (Barcaccia et al, 1999). To detect groups of linked markers, a minimum LOD score of 3.0 and a maximum recombination frequency of $r=0.35$ was applied to both methods. Map distances, expressed in centiMorgans (cM), were calculated by the Kosambi's mapping function (Kosambi, 1944).

\section{Results}

\section{Polymorphism type and degree}

A total of 970 AFLP marker loci were detected in the $F_{1}$ progenies using the 15 primer combinations. As many as 745 AFLP marker alleles $(76.8 \%)$ were polymorphic between parental species (20.3 polymorphisms per primer combination), whereas 225 marker alleles (23.2\%) were shared. Of the AFLP polymorphisms, 489 were $S$. alba-specific (50.4\% of total marker alleles) and 256 S. fragilis-specific ( $26.4 \%$ of total marker alleles). A maximum of 33 polymorphic fragments were obtained with the primer combination $P s t+\mathrm{AT} / M s e+\mathrm{CAG}$, and a minimum of 14 with the primer combination Eco+CC/ Mse+AGT.

A total of 152 SAMPL marker loci were detected using two primer combinations. Of these, 27 (17.7\%) were shared between parents, while 125 (82.2\%) were polymorphic between parental species. As many as 79 SAMPL marker alleles $(52.0 \%$ of total) were $S$. albaspecific and 46 marker alleles $(30.3 \%)$ were $S$. fragilisspecific. The primer combination As $2 / M s e+A A$ generated 30 polymorphic fragments. Primer As1 was unable to find homologous binding sites so that fingerprints with a reliable number of strong amplification products could be produced.

Overall, of the 870 markers polymorphic between parents in both crosses, as many as 568 (65.3\%) were from S. alba and $302(34.7 \%)$ were from S. fragilis. The difference between the numbers of $S$. alba-specific and $S$. fragilis-specific markers, as assessed by a contingency test, was highly significant $\left(\chi^{2}=30.4^{* * *}, 1 \mathrm{df}\right)$. Within each species, the contribution of maternal and paternal polymorphisms was comparable with both the AFLP (266 vs 223 in S. alba and 109 vs 147 in S. fragilis) and SAMPL (40 vs 39 in S. alba and 23 vs 23 in S. fragilis) marker systems.

\section{Segregation pattern analysis and single-dose marker detection}

$\chi^{2}$ analyses for various segregation ratios (from 1:4 up to $4: 1$, presence vs absence) were performed. The most represented segregation ratios ranged from 0.75 to 1.25 as expected for SDMs, with the mean ratios of 0.99 for $S$. alba and 1.08 for S. fragilis markers (Figure 1). A very low proportion of markers with skewed segregation patterns was observed in both species (Table 2).

A total of $364(41.8 \%)$ marker alleles of the 870 that were polymorphic between parents were shown to segregate in the $F_{1}$ progenies. As many as 292 of these $(80.2 \%)$ were SDMs. In particular, of the 305 segregating AFLPs, $242(79.3 \%)$ were inherited as parent-specific SDMs (163 from S. alba and 79 from S. fragilis). This corresponds, on average, to 16.2 (8.1 paternal and 8.1 maternal) SDMs per primer combination. Moreover, 50 of the $59(84.7 \%)$ segregating SAMPLs were inherited as parent-specific SDMs (31 from $S$. alba and 19 from $S$. fragilis) with an average of 25 (15.5 paternal and 9.5 maternal) SDMs per primer combination. There were no significant differences in the relative contribution of the two types of molecular markers to the various SDM segregation classes (data not shown). On the whole, the mean number of parent-specific SDM polymorphisms supplied per assay by the two marker systems did not differ significantly $\left(\chi^{2}=0.073,1 \mathrm{df}\right.$, $P<0.05)$.

Overall, 194 S. alba (81 paternal and 113 maternal) and 98 S. fragilis (62 paternal and 36 maternal) SDMs polymorphic between parents segregated in a 1:1 Mendelian fashion $(P \geqslant 0.05)$, while 22 SDMs shared between parents segregated in a 3:1 Mendelian fashion $(P \geqslant 0.05)$. Of the remaining marker loci analysed, only 29 paternal (24 AFLPs and 5 SAMPLs) and 19 maternal (18 AFLPs and 1 SAMPLs) were markedly distorted $(P<0.01)$. Segregation distortion of $S$. alba marker loci (34 out of $239,14.2 \%$ ) did not differ significantly $\left(\chi^{2}=0.419, \mathrm{df}=1\right)$ from that of the $S$. fragilis (14 out of $125,11.2 \%)$. 


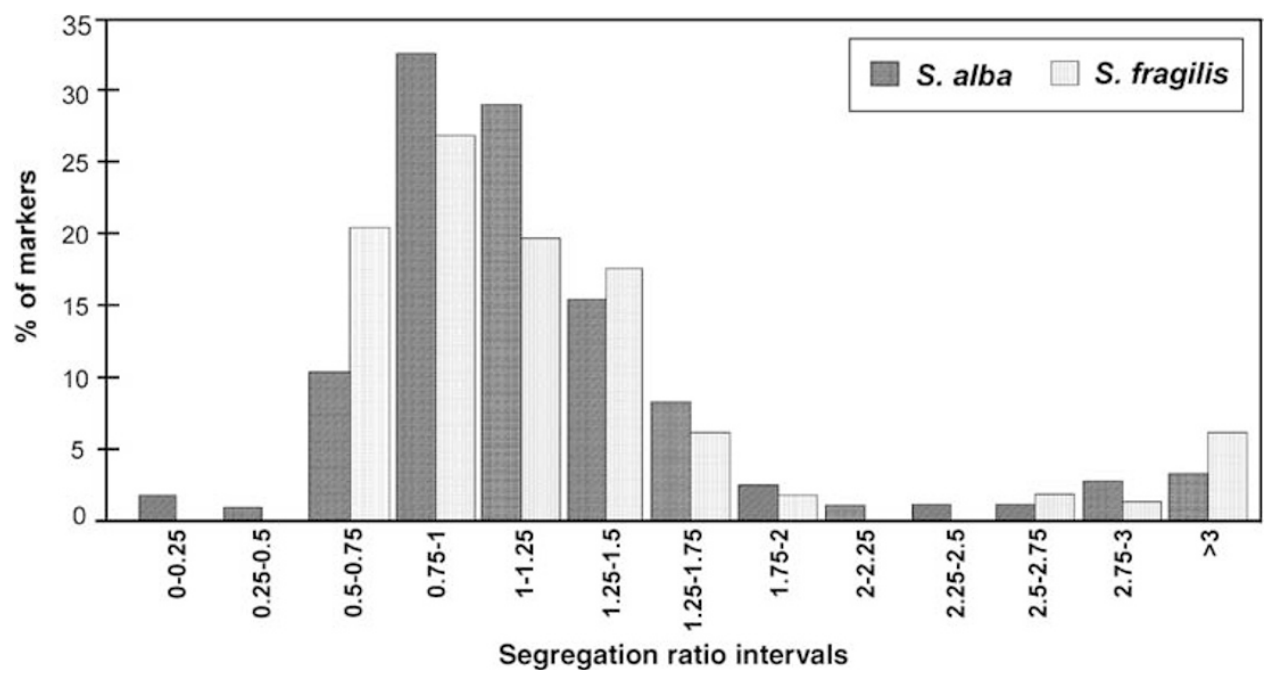

Figure 1 Distribution of segregation ratios (presence vs absence) of Salix alba (239) and S. fragilis (125) markers in the $\mathrm{F}_{1}$ progenies. The 364 markers polymorphic between parental plants were detected with 15 AFLP and two SAMPL primer combinations. Markers with a ratio greater than 2.5:1 were not considered SDMs and thus not used for linkage mapping.

Table 2 Information on the inheritance patterns observed for single- and multiple-dose marker alleles in Salix alba and S. frangilis

\begin{tabular}{ccccc} 
& Segregating markers $(P>0.05)^{a}$ & & $\begin{array}{c}\text { Distorted } \\
\text { markers }\end{array}$ & $\begin{array}{c}\text { Monomorphic } \\
\text { markers }\end{array}$ \\
\cline { 1 - 3 } $1: 1$ ratio & $3: 1$ ratio & $5: 1$ ratio & $11: 1$ ratio &
\end{tabular}

Marker polymorphic

between parents

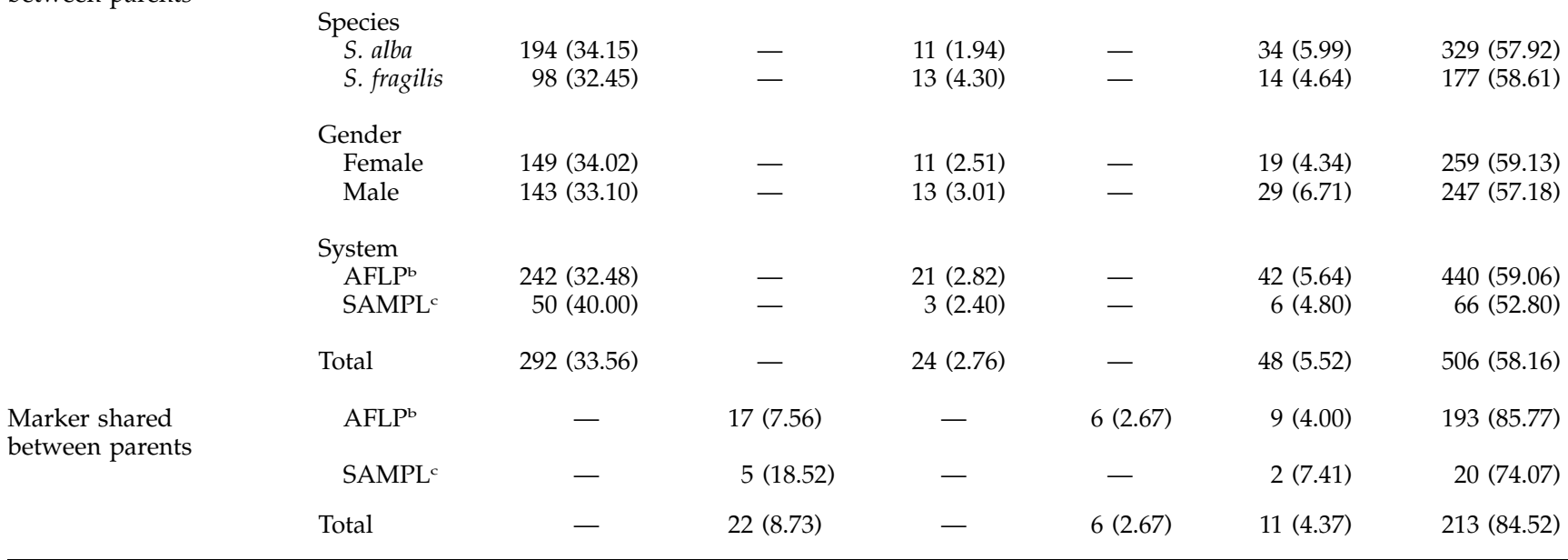

${ }^{\mathrm{a}}$ The total number of loci and the relative percentage (within parentheses) are presented for segregating markers. ${ }^{\mathrm{b}} \mathrm{AFLP}$ marker loci assayed using 15 primer combinations. 'SAMPL marker loci for two primer combinations.

While 1:1 and 3:1 are the only segregation ratios expected for an allotetraploid condition and detectable uniquely with SDMs, additional segregation patterns in autotetraploid genomes may also be identified using DDMs. Of the 870 and 252 marker loci, respectively, polymorphic and shared between parents, only 24 (2.8\%) plus six $(2.4 \%)$ showed a segregation attributable to DDMs on the basis of $\chi^{2}$ values. Segregation patterns observed for these marker loci correspond to segregation ratios, respectively, of 5:1 and 11:1 (presence vs absence) expected in duplex by nulliplex and duplex by simplex crosses (Table 2).

\section{Chromosome pairing behaviour}

According to the Mather test, maternal and paternal SDMs were ordered in 55 cosegregation groups. SDMs of each pair of cosegregation group were then tested for the linkage phase on the basis of parental vs recombinant patterns. The number of SDMs linked in coupling and the number of those linked in repulsion calculated for each pairwise comparison were then assayed to fit the 1:1 expected ratio ( $\mathrm{Wu}$ et al, 1992). Of the $S$. alba cosegregation groups, 14 out of the 19 maternal linkage groups and 13 out of the 16 paternal linkage groups showed a nonsignificant deviation $(P \leqslant 0.05)$, suggesting 
Table 3 Linkage phase of marker loci belonging to S. alba and $S$. fragilis cosegregating female and male marker loci groups

\begin{tabular}{|c|c|c|c|c|c|c|}
\hline \multirow{2}{*}{$\begin{array}{l}\text { Species } \\
\text { gender }\end{array}$} & \multirow{2}{*}{$\begin{array}{l}\text { Linkage } \\
\text { group }\end{array}$} & \multicolumn{4}{|c|}{ Linkage phase $e^{a}$} & \multirow{2}{*}{$\begin{array}{c}\text { Chromosome } \\
\text { pairing }\end{array}$} \\
\hline & & Coupling & Repulsion & $\chi^{2}$ & $P$ & \\
\hline \multirow{19}{*}{$\begin{array}{l}\text { S. alba } \\
\text { (female) }\end{array}$} & 1 & 149 & 105 & 7.62 & $* *$ & $\mathrm{r}$ \\
\hline & 2 & 129 & 104 & 2.68 & NS & $p$ \\
\hline & 3 & 115 & 88 & 3.59 & NS & $\mathrm{p}$ \\
\hline & 4 & 50 & 71 & 3.64 & NS & $\mathrm{p}$ \\
\hline & 5 & 38 & 25 & 2.68 & NS & $\mathrm{p}$ \\
\hline & 6 & 43 & 23 & 6.06 & $*$ & $\mathrm{r}$ \\
\hline & 7 & 74 & 36 & 13.13 & $* * *$ & $\mathrm{r}$ \\
\hline & 8 & 25 & 15 & 2.50 & NS & $\mathrm{p}$ \\
\hline & 9 & 22 & 18 & 0.40 & NS & $\mathrm{p}$ \\
\hline & 10 & 20 & 19 & 0.03 & NS & $\mathrm{p}$ \\
\hline & 11 & 15 & 16 & 0.03 & NS & $\mathrm{p}$ \\
\hline & 12 & 17 & 9 & 2.46 & NS & p \\
\hline & 13 & 36 & 46 & 1.22 & NS & $\mathrm{p}$ \\
\hline & 14 & 74 & 47 & 6.02 & $*$ & $\mathrm{r}$ \\
\hline & 15 & 24 & 14 & 2.63 & NS & $p$ \\
\hline & 16 & 29 & 27 & 0.07 & NS & $\mathrm{p}$ \\
\hline & 17 & 37 & 14 & 10.37 & $* * *$ & $\mathrm{r}$ \\
\hline & 18 & 15 & 26 & 2.95 & NS & $\mathrm{p}$ \\
\hline & 19 & 37 & 25 & 2.32 & NS & $\mathrm{p}$ \\
\hline \multirow{16}{*}{$\begin{array}{l}\text { S. alba } \\
\text { (male) }\end{array}$} & 1 & 56 & 36 & 4.35 & * & $\mathrm{r}$ \\
\hline & 2 & 43 & 17 & 11.27 & $* * *$ & $\mathrm{r}$ \\
\hline & 3 & 22 & 12 & 2.94 & NS & $\mathrm{p}$ \\
\hline & 4 & 21 & 16 & 0.68 & NS & $p$ \\
\hline & 5 & 17 & 22 & 0.64 & NS & $\mathrm{p}$ \\
\hline & 6 & 43 & 44 & 0.01 & NS & $\mathrm{p}$ \\
\hline & 7 & 44 & 49 & 0.27 & NS & $\mathrm{p}$ \\
\hline & 8 & 16 & 13 & 0.31 & NS & $\mathrm{p}$ \\
\hline & 9 & 22 & 22 & 0.00 & NS & p \\
\hline & 10 & 19 & 24 & 0.58 & NS & $\mathrm{p}$ \\
\hline & 11 & 20 & 20 & 0.00 & NS & $\mathrm{p}$ \\
\hline & 12 & 15 & 16 & 0.03 & NS & $\mathrm{p}$ \\
\hline & 13 & 17 & 9 & 2.46 & NS & $\mathrm{p}$ \\
\hline & 14 & 37 & 14 & 10.37 & $* * *$ & $\mathrm{r}$ \\
\hline & 15 & 22 & 18 & 0.40 & NS & $p$ \\
\hline & 16 & 10 & 6 & 1.00 & NS & $\mathrm{p}$ \\
\hline \multirow{6}{*}{$\begin{array}{l}\text { S. fragilis } \\
\text { (female) }\end{array}$} & 1 & 6 & 10 & 1.00 & NS & $\mathrm{p}$ \\
\hline & 2 & 6 & 2 & 2.00 & NS & p \\
\hline & 3 & 12 & 14 & 0.15 & NS & p \\
\hline & 4 & 4 & 6 & 0.40 & NS & $\mathrm{p}$ \\
\hline & 5 & 6 & 11 & 1.47 & NS & $\mathrm{p}$ \\
\hline & 6 & 2 & 0 & 2.00 & NS & $\mathrm{p}$ \\
\hline \multirow{14}{*}{$\begin{array}{l}\text { S. fragilis } \\
\text { (male) }\end{array}$} & 1 & 2 & 10 & 5.33 & * & $\mathrm{r}$ \\
\hline & 2 & 10 & 6 & 1.00 & NS & $\mathrm{p}$ \\
\hline & 3 & 14 & 22 & 1.78 & NS & $p$ \\
\hline & 4 & 6 & 12 & 2.00 & NS & $\mathrm{p}$ \\
\hline & 5 & 26 & 16 & 2.38 & NS & $\mathrm{p}$ \\
\hline & 6 & 16 & 16 & 0.00 & NS & p \\
\hline & 7 & 12 & 8 & 0.80 & NS & $\mathrm{p}$ \\
\hline & 8 & 16 & 16 & 0.00 & NS & $\mathrm{p}$ \\
\hline & 9 & 14 & 20 & 1.06 & NS & $\mathrm{p}$ \\
\hline & 10 & 14 & 20 & 1.06 & NS & $p$ \\
\hline & 11 & 18 & 8 & 3.85 & NS & $\mathrm{p}$ \\
\hline & 12 & 12 & 8 & 0.80 & NS & $\mathrm{p}$ \\
\hline & 13 & 8 & 24 & 8.00 & $* * *$ & $\mathrm{r}$ \\
\hline & 14 & 10 & 0 & 10.00 & $* * *$ & $\mathrm{r}$ \\
\hline
\end{tabular}

aNumber of markers linked in coupling and repulsion over all pairwise comparisons. ${ }^{\mathrm{b}} \mathrm{p}$, preferential; $\mathrm{r}$, random.

preferential chromosome pairing behaviour. In S. fragilis, of the cosegregation groups detected, only three of 20 maternal and paternal linkage groups constructed showed significant deviations $(P \leqslant 0.01)$, again supporting preferential chromosome pairing behaviour (Table 3 ).
The hypothesis of preferential chromosome pairing was tested by performing a linkage analysis using the $\mathrm{CP}$ mapping population option, which allows markers in both the coupling and repulsion phases to be linked in allopolyploid species as in diploids. Additional markers were mapped only in those groups indicated by the Mather test as being affected by preferential chromosome pairing. These additional alleles were mapped in repulsion alongside a framework of marker alleles in coupling, without changing either distances or order of markers previously identified with the $\mathrm{BC}_{1}$ mapping population option (Figure 2). No additional repulsion marker alleles were added to the original framework of coupling markers for the groups that the Mather test had indicated as affected by random chromosome pairing (Wu et al, 1992). Several pairs of marker alleles from single-parents that exhibited codominant inheritance patterns were detected (Figure 3).

\section{Linkage map construction}

Four linkage maps were obtained using JoinMap 3.0 by employing the CP option. In S. alba, 67 out of 113 maternal SDMs (53 AFLPs and 14 SAMPLs) were mapped to 19 linkage groups (Figure 4a) covering $708 \mathrm{cM}$ (mean, $37.3 \mathrm{cM}$ and 3.6 markers per group); 42 of the 81 paternal SDMs (mean, 36 AFLPs and six SAMPLs) were mapped to 16 linkage groups (Figure $4 \mathrm{~b}$ ) covering $339 \mathrm{cM}$ (mean, $21.2 \mathrm{cM}$ and 3.0 markers per group).

In S. fragilis, 33 out of 62 paternal SDMs (26 AFLPs and seven SAMPLs) were mapped in 14 linkage groups (Figure 5a) covering $321 \mathrm{cM}$ (mean, $22.9 \mathrm{cM}$ and 2.4 markers per group); 13 of the 36 maternal SDMs (seven AFLPs and six SAMPLs) were mapped to six linkage groups (Figure $5 \mathrm{~b}$ ) covering $98 \mathrm{cM}$ (mean, $16.3 \mathrm{cM}$ and 2.2 markers per group).

By applying an LOD score of 2.0, 12 additional SDMs of both male (six AFLPs) and female (five AFLPs and one SAMPL) S. alba were found to belong to the linkage groups detected. These SDMs have been added in the two maps by positioning them next to the nearest framework markers because the 'ripple' function was unable to confirm the final order of loci. The paternal maps included $59.3 \%$ (S. alba) and $40.7 \%$ (S. fragilis) of the paternal SDMs, while the maternal maps included $84.9 \%$ (S. alba) and $15.1 \%$ (S. fragilis) of the maternal SDMs. Overall, $62.4 \%$ of the S. alba SDMs and $46.9 \%$ of the $S$. fragilis SDMs segregating 1:1 were mapped. No significant difference $\left(\chi^{2}=2.37 ; P \leqslant 0.05\right)$ was found between the proportion of AFLP and SAMPL marker alleles mapped among those scored as segregating 1:1 in the $F_{1}$ populations (ie 133 out of $242,54.9 \%$, and 34 out of 50 , $68.0 \%)$

\section{Discussion}

Allotetraploids are those polyploids that have arisen through the processes of interspecific hybridisation and chromosome doubling, whereas autotetraploids are those polyploids that have arisen from conspecific parents (Soltis and Soltis, 2000). The former are characterised by fixed (ie nonsegregating) heterozygosity, resulting from the combination of divergent parental genomes, such that bivalent formation occurs at meiosis and disomic inheritance operates at each locus. The latter 


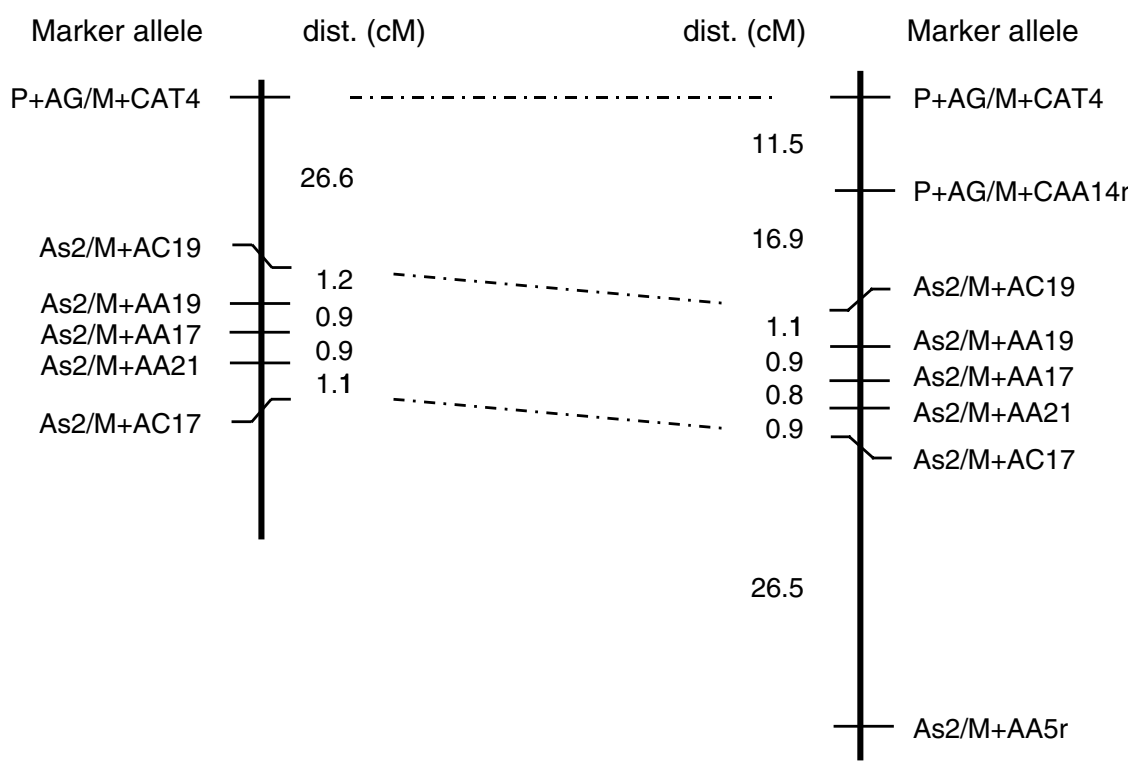

Figure 2 Comparison of linkage group 3 of female Salix alba as obtained with the $\mathrm{BC}_{1}$ and $\mathrm{CP}$ mapping population options. Marker loci mapped in repulsion are prefixed by an ' $r$ ' and are linked between them in coupling $\left(\chi^{2}=14.34\right)$.

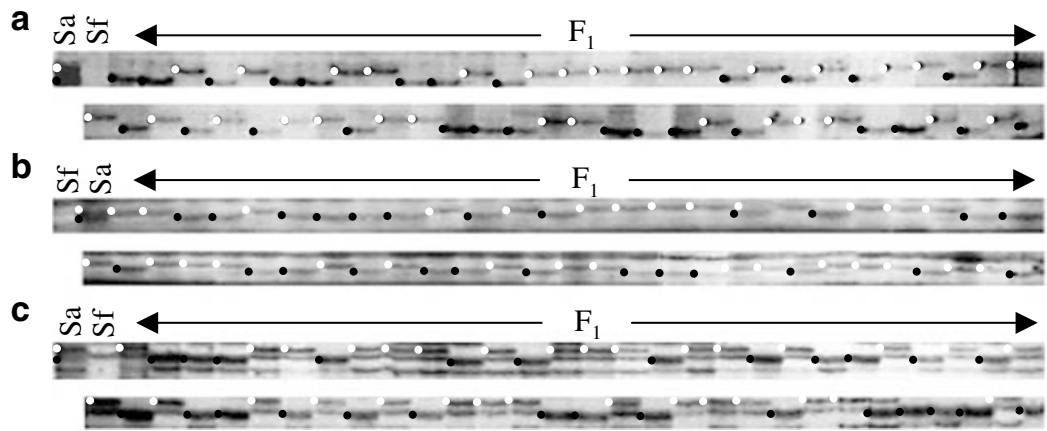

Figure 3 Examples of segregation patterns of codominant marker loci detected by AFLP primer combinations Pst+AT/Mse+CCA (a), $E c o+C C / M s e+A T C(b)$, and Pst+AG/Mse+CAC (c). Black and white bullets indicate single marker alleles segregating from the female (a, c) and male (b) S. alba parents.

usually exhibits multivalent formation at meiosis and are characterised by polysomic inheritance, when more than two different alleles occur at each locus. Although the analysis of pairing behaviour of tetraploid willow trees could help explain the genomic constitution of willows, cytological observations in this species are difficult to perform owing to the high chromosome number and small chromosome size.

Verification of segregation patterns and construction of linkage maps for polyploids can have great implications not only for marker-assisted selection, but also for polyploid genome classification, evolution and population genetic studies. Although the frequency of polyploidy has been estimated to be as much as $70 \%$ of all Angiosperms (Masterson, 1994), linkage mapping efforts in polyploid crops and forest trees are scanty. During the last few years, a few theoretical, simulation and explorative studies have been performed to estimate linkage in polyploids (Sorrells, 1992; Wu et al, 1992, 2001;
Yu and Pauls, 1993; Xu et al, 1995; Hackett et al, 1998; Qu and Hancock, 2001). In addition, both RFLP- and PCRbased markers have been exploited to develop linkage maps of tetraploid species such as potato (Jacobs et al, 1995), sugarcane (Grivet et al, 1996), alfalfa (Brouwer and Osborn, 1999), cotton (Brubaker et al, 1999) and rose (Rajapakse et al, 2001). In general, however, the construction of genetic maps of polyploid species has lagged behind that for diploid species because the statistical methods are far more complicated than for diploids, and little is known about the genomic constitution and pairing behaviour of most polyploids. Moreover, large segregating populations are needed to obtain reliable genetic distance estimates.

A crucial step in constructing genetic maps in polyploid species is the determination of marker dosage (Porceddu et al, 2002). In tetraploid willow, this was done by counting the proportion of plants in the progeny of a pseudotestcross that exhibited a given marker allele and 
testing this proportion against those expected for various marker dosages. Of the total molecular markers found to be polymorphic between parents, more than $58 \%$ did not

a

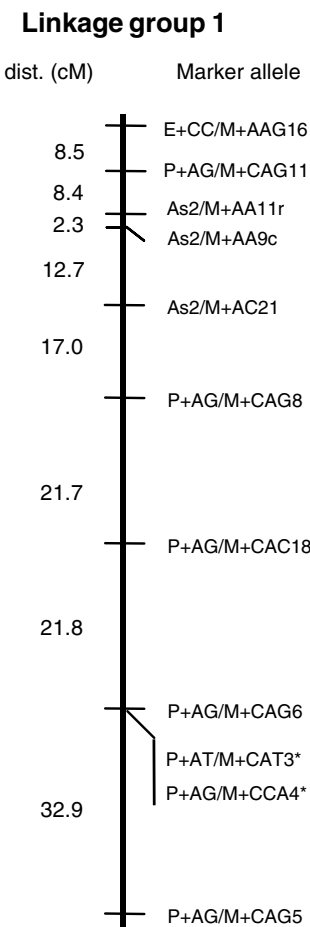

$$
\begin{aligned}
& \begin{array}{l}
\text { Linkage group } 8 \\
\text { dist. (cM) } \quad \text { Marker allele } \\
\qquad 1.0 \\
\text { P+AG/M+CAA13 } \\
\text { P+AG/M+CAT10 }
\end{array}
\end{aligned}
$$

Linkage group 12

dist. (cM) Marker allele

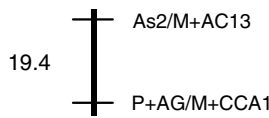

\section{Linkage group 16}

dist. (cM) Marker allele

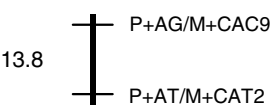

\section{Linkage group 2}

dist. (cM) Marker allele

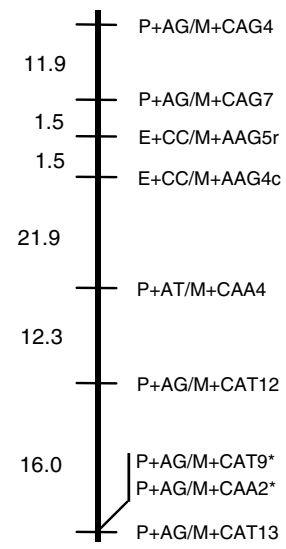

\section{Linkage group 5}

dist. (cM) Marker allele

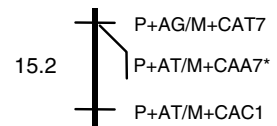

\section{Linkage group 9}

dist. (cM) Marker allele

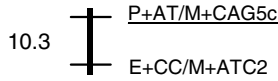

\section{Linkage group 13}

dist. (CM) Marker allele

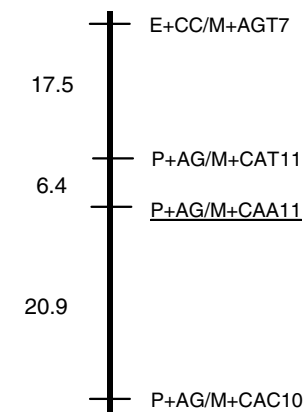

\section{Linkage group 17}

dist. (CM) Marker allele

$$
7.5 \text { † } \mathrm{P}+\mathrm{AT} / \mathrm{M}+\mathrm{AT} / \mathrm{CAT}+\mathrm{CAT} 1
$$

segregate in the $F_{1}$ progenies as a consequence of multiple-dose alleles at the loci being tested, while about one-third $(33.6 \%)$ were inherited as SDMs. The fact that,

\section{Linkage group 3}

dist. (cM) Marker allele

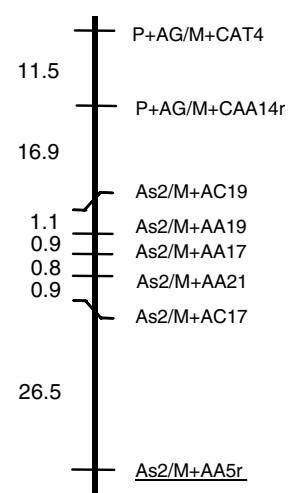

\section{Linkage group 6}

dist. (cM) Marker allele

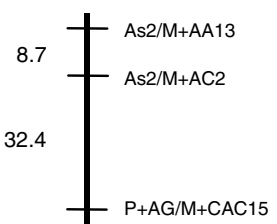

\section{Linkage group 10}

dist. (cM) Marker allele

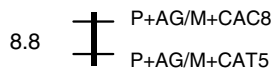

\section{Linkage group 14}

dist. (cM) Marker allele

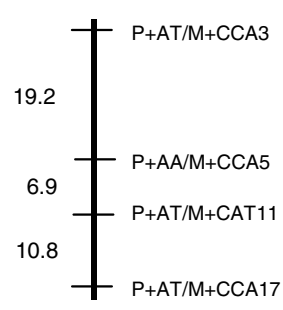

\section{Linkage group 18}

dist. (cM) Marker allele

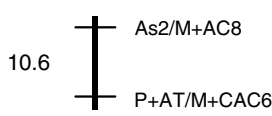

\section{Linkage group 4}

dist. (cM) Marker allele

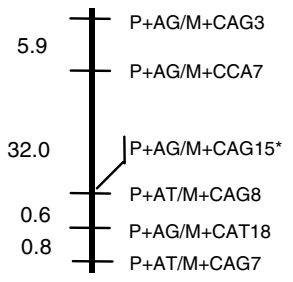

\section{Linkage group 7}

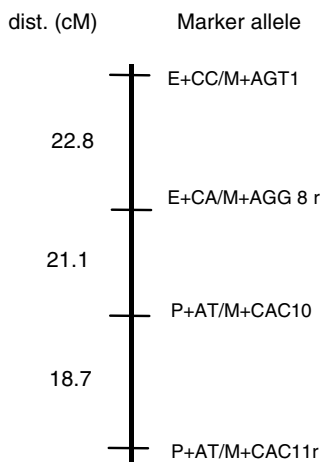

\section{Linkage group 11}

$$
\begin{array}{cc}
\text { dist. (cM) } & \begin{array}{c}
\text { Marker allele } \\
\end{array} 28.5 \\
& \text { P+AT/M+CCA24 } \\
\text { P+AT/M+CAA5 }
\end{array}
$$

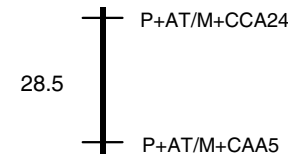

\section{Linkage group 15}

dist. (cM) Marker allele

$$
25.2 \underbrace{}_{{ }_{P+A G / M+C A G 2}}
$$

\section{Linkage group 19}

dist. (cM) Marker allele

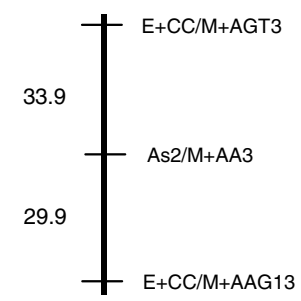

Figure 4 Genetic linkage maps of female (a) and male (b) Salix alba based upon AFLP and SAMPL markers. The framework map showing the linear order and relative distances in cM between markers was constructed according to a multipoint analysis with an LOD score of at least 3 and a recombination frequency of 0.35 . Marker loci are identified by the primer combination ( $\mathrm{P}, \mathrm{E}$ and $\mathrm{M}$ stand for Pst, Eco and Mse primers) and by a number indicating the relative position detected in the gel lane. Codominant loci are flanked by a ' $c$ ' and loci mapped in repulsion phase by an ' $r$ '. Marker loci shared by the male and female maps are underlined. Marker loci marked with an asterisk were mapped with an LOD of 2 and are positioned next to the nearest framework marker. 
b
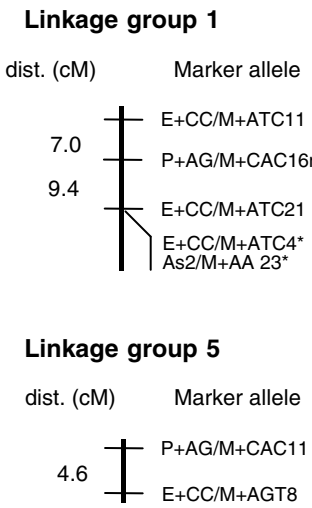

Linkage group 9

dist. (cM) Marker allele

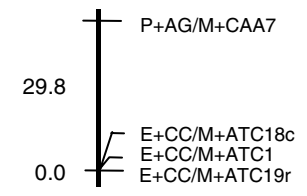

Linkage group 13

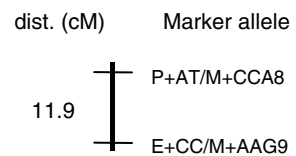

Linkage group 2

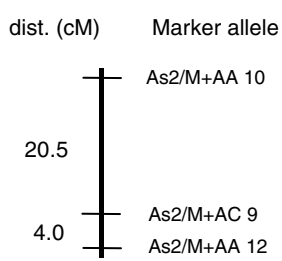

Linkage group 6

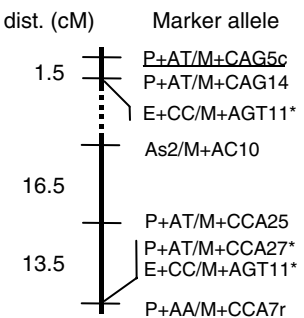

Linkage group 10

dist. (cM) Marker allele

$\begin{array}{lll}21.7 & \text { E+CC/M+ATC5 } \\ 16.7 & \text { P+AT/M+CAG27 } \\ & \text { P+AT/M+CAG21 }\end{array}$

Linkage group 14

dist. (cM) Marker allele

$9.1 \underset{\text { P+AA/M+CCA8 }}{\text { P+AT/M+CAG13 }}$
Linkage group 3

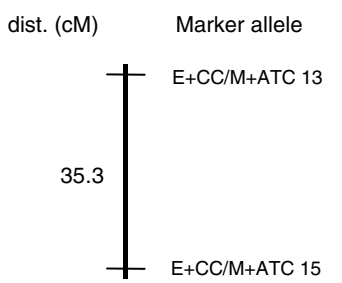

Linkage group 7

dist. (cM) Marker allele

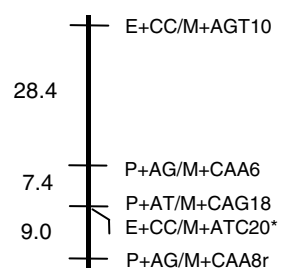

Linkage group 11

dist. (cM) Marker allele

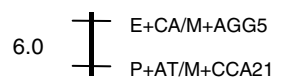

\section{Linkage group 15}

dist. (cM) Marker allele

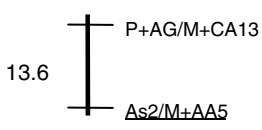

\section{Linkage group 4}

dist. (cM) Marker allele

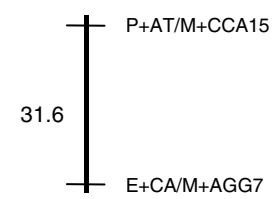

Linkage group 8

dist. (cM) Marker allele

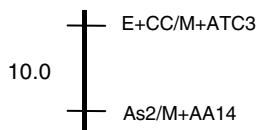

Linkage group 12

dist. (cM) Marker allele

$$
21.5{ }^{\mathrm{P}+\mathrm{AG} / \mathrm{M}+\mathrm{CAA} 10}
$$

Linkage group 16

dist. (cM) Marker allele

$10.4 \underset{{ }_{P}+A G / M+C A C 9}{P+A G / M+C A A 11}$

Figure 4 Continued.

of the 1122 marker loci completely identified in the two male and female parents, the vast majority $(77.5 \%)$ were proven to be polymorphic and as few as $22.5 \%$ were shared between parental species, highlights that $S$. alba and $S$. fragilis individuals are genotypically highly differentiated. If it is true that derived species are highly rarer and contain fewer alleles, the highly significant difference between $S$. alba-specific and $S$. fragilis-specific markers found in both parental combinations (mean, 65.3 vs $34.7 \%$, respectively) supports the phylogenetic hypothesis formulated by Triest et al (1999), that $S$. fragilis is a derived from $S$. alba-like progenitors. Compared to allozyme data from Northern American diploid species (Aravanopoulos et al, 1993), polyploid species of willows showed a similar degree of heterozygosity. On the other hand, in the light of the new findings of this paper, nonvariable duplicated genes (eg for PGM loci) reported for tetraploid willows by Triest et al (1998) could be interpreted as a result of the allopolyploid origin of these species.

The $S$. alba $\times$ S. fragilis $\mathrm{F}_{1}$ populations obtained by crossing two unrelated parents were studied assuming that a noninbred population would not only provide an effective strategy for limiting segregation distortion, but would also give higher degrees of polymorphic loci and better estimates of linkage distances (Barcaccia et al, 1999, 2000a). In fact, about $80 \%$ of polymorphic SDMs (292 out of 364) were inherited according to the Mendelian segregation ratio of $1: 1$ (for $P \geqslant 0.05$ ). As many as 50 $(22+22+6)$ single-parent SDMs of genomic loci that shared marker alleles between parents showed nonsignificant 3:1 (presence vs absence) segregation patterns. Some of these latter marker alleles segregating from both the maternal and paternal parents will be essential for integrating the linkage groups of each species.

In polyploid species, chromosome pairing may be preferential and/or random suggesting, an allopolyploid, autopolyploid or mixed genomic origin, respectively. The pairwise analysis of cosegregation groups linked in repulsion indicated that most of the willow chromosomes pair preferentially in $S$. alba-S. fragilis interspecific hybrids. This finding supports a low genomic affinity between these two species and suggests that the recombination potential between the two species is limited. Analysis of other genotypes would show whether this supposition is valid and applies to a wide range of germplasm sources. Although hybridisation can take place between $S$. alba and $S$. fragilis giving rise to fertile interspecific progenies, molecular data collected in this study suggest that the low genomic exchange would occur, causing both species to maintain separate gene pools. Fixation indices for several allozyme loci indicated values deviating highly from an equilibrium when considering $S$. alba and $S$. fragilis as a single species complex, supporting the lack of gene exchange between these species (Triest et al, 1999). The fixation indices 


\section{a}

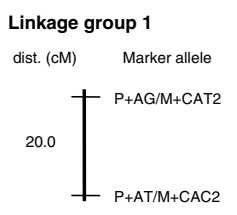

Linkage group 5

dist. (CM) Marker allele

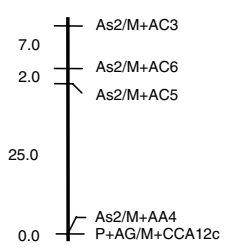

Linkage group 9

dist. (cM) Marker allele

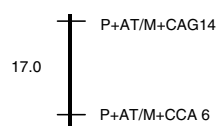

Linkage group 14

dist. (cM) Marker allele

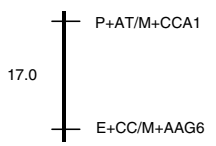

\section{b}

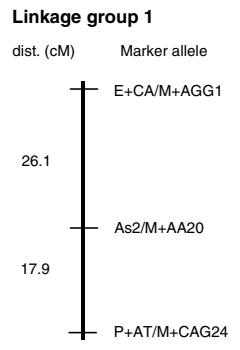

Linkage group 2

dist. (CM) Marker allele

29.0 † ${ }^{\mathrm{E}+\mathrm{A}+\mathrm{AT} / \mathrm{M}+\mathrm{M}+\mathrm{CAC} 12}$

Linkage group 6

dist. (CM) Marker allele

$24.0 \overbrace{}^{\mathrm{P}+\mathrm{AG} / \mathrm{M}+\mathrm{CCA} 8}$

- As2/M+AA1

9.0

I As2/M+AC11

Linkage group10

dist. (CM) Marker allele

$20.1 \overbrace{\text { E+CC/M+ATC8 }}^{\text {P+AT/M+CAG16 }}$
Linkage group 3

dist. (CM) Marker allele

$22.0 \overbrace{\text { E+CC/M+AAG19 }}^{\mathrm{P}+A T / M+C C A 10}$

Linkage group 7

dist. (CM) Marker allele

$6.0 \coprod^{\mathrm{P}+\mathrm{AT} / \mathrm{M}+\mathrm{AT} / \mathrm{M}+\mathrm{CAG} 1}$

Linkage group 11

dist. (cM) Marker allele

\rfloor$^{15.0}{ }^{\mathrm{P}+\mathrm{AG} / \mathrm{M}+\mathrm{AG} / \mathrm{M}+\mathrm{CAG} 16}$

Linkage group 12

dist. (cM) Marker allele

$15.0 \overbrace{P+A T / M+C A C 7}^{P+A T / M+C A G 4}$
Linkage group 4

dist. (cM) Marker allele

$17.0 \overbrace{P+A T / M+C A A 2}^{P+A G / M+C A C 3}$

Linkage group 8

dist. (CM) Marker allele

†

Linkage group 13

dist. (CM) Marker allele

$\overbrace{}^{{ }_{P+A G / M+C C A 11}+A G / M+C A G 13}$

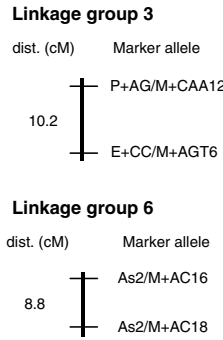

Linkage group 4

dist. (cM) Marker allele

$\prod_{11.8} \underline{\mathrm{As} 2 / \mathrm{M}+\mathrm{AA} 2}$

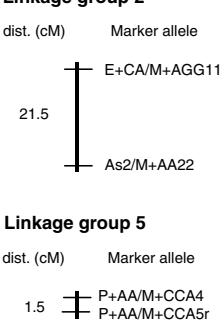

$+{ }_{\text {As2 } 2 M+A C 18}$

Figure 5 Genetic linkage map of male (a) and female (b) Salix fragilis (details in legend to Figure 4).

deviated even more from an equilibrium when considering both species as separate reproductive units. In the view of the recent findings, this is an additional indication of the low recombination potential between the two species.

It has been suggested that the ratios of coupling-torepulsion-phase-linked markers can be used to distinguish between allopolyploids and autopolyploids, because repulsion-phase linkages are much more difficult to detect in autopolyploids with polysomic inheritance than allopolyploids with disomic inheritance $(\mathrm{Qu}$ and Hancock, 2001). Wu et al (1992) have reported that when homologous chromosomes pair randomly (autopolyploids), the power of detection of repulsion-phase linkages is very low. In particular, with a population size of 67 and a confidence level of $5 \%$, the maximum detectable distance in autotetraploids is $7.4 \mathrm{cM}$ and no linkages can be detected at higher ploidy levels. In contrast, when chromosomes pair specifically at meiosis (allopolyploids), the detection power of coupling and repulsion linkages is the same. As a consequence, in allotetraploids, if the chromosomes pair systematically and specifically, SDMs assigned to the cosegregation group will be in repulsion with a comparable number of cosegregating SDMs, whereas their number will be much lower if the chromosomes pair randomly.

For most cosegregation groups, a comparable number of markers linked in coupling and repulsion was identified. This finding suggests that most basic chromosomes pair preferentially as happens for allotetraploid species with disomic inheritance. This is further supported by the detection of several pairs of marker alleles of single parents showing codominant inheritance patterns. Previous work (Triest et al, 1998), involving progeny tests for monomeric enzymes (eg LAP and PGM) in inter- and intraspecific crosses of both willow species, allowed rejection of the hypothesis of autotetraploidy in most progenies.

With dominant markers, segregation patterns fitting the 1:1 and 3:1 presence $v$ s absence ratios are expected whether parental genome constitution is of the autotetraploid or allotetraploid type. However, in this study, 
a number of genomic loci showing segregation patterns detectable uniquely under tetrasomic inheritance (eg 5:1 and 11:1 presence $v$ s absence) were also found. This result indicates that tetraploidy in willows may have arisen from partially related diploid ancestral parents with some homologous chromosomal regions. While allopolyploids and autopolyploids are two extremes of polyploids, a number of polyploid taxa actually represent intermediate stages displaying a combination of both allopolyploid and autopolyploid pairing behaviour (Wu et al, 1992). The mosaic of disomy and tetrasomy at various loci might be a general mechanism underlying the inheritance of many tetraploids, including S. alba and S. fragilis.

Unlike RFLPs and SSRs, which might identify cosegregation groups on the basis of allellism, AFLPs and SAMPLs as dominant marker systems have the obvious disadvantage of making it difficult to identify homologous cosegregation groups in order to construct genetic linkage maps in polyploids. This disadvantage of AFLPs and SAMPLs is, however, partially offset by the very high multiplex-ratio as well as the high-yield chromosome-specific markers. Interestingly, the AFLP technique with PstI/MseI primer combinations was the most efficient in detecting SDMs. The higher incidence of restriction sites and/or their different genomic distribution probably explain this finding. Currently, we are cloning and characterising microsatellites (ie TC, CA and GCT repeats) to be used as codominant bridge loci for the construction of an integrated interspecific map of willow.

\section{Acknowledgements}

We thank Prof. Paolo Paiero, TESAF, University of Padova (Italy) for providing the willow clones.

\section{References}

Alstrom-Rapaport C, Lascoux M, Wang YC, Roberts G, Tuskan GA (1998). Identification of a RAPD marker linked to sex determination in the basket willow (Salix viminalis L.). J Hered 89: 44-49.

Aravanopoulos F, Szuffa L, Chong K (1993). The genetic basis of enzymatic variation in Salix exigua. Hereditas 119: 77-88.

Barcaccia G, Mazzucato A, Albertini E, Zethof J, Gerats T, Pezzotti M, Falcinelli M (1998). Inheritance of parthenogenesis in Poa pratensis L.: auxin test and AFLP linkage analysis support monogenic control. Theor Appl Genet 97: 74-82.

Barcaccia G, Albertini E, Tavoletti S, Falcinelli M, Veronesi F (1999). AFLP fingerprinting in Medicago spp.: its development and application in linkage mapping. Plant Breed 118: 335-340.

Barcaccia G, Paiero P, Lucchin M (2000a). Investigating genetic and molecular relationships among species of the genus Salix L. belonging to section Fragiles Fr. Monti Boschi 1: 52-58 (in Italian with English summary).

Barcaccia G, Albertini E, Rosellini D, Tavoletti S, Veronesi F (2000b). Inheritance and mapping of $2 n$ egg production in diploid alfalfa. Genome 43: 1-10.

Beismann H, Barker JHA, Karp A, Speck T (1997). AFLP analysis sheds light on distribution of two Salix species and their hybrid along a natural gradient. Mol Ecol 6: 989-993.

Brouwer DJ, Osborn TC (1999). A molecular linkage map of tetraploid alfalfa (Medicago sativa L.). Theor Appl Genet 99: 1194-1200.
Brubaker CL, Paterson AH, Wendel JF (1999). Comparative genetic mapping of allotetraploid cotton and its diploid progenitors. Genome 42: 184-203.

De Bondt R (1996). Role of Genetic Diversity and Identity in Replantations - Case Study of Salix sp. M.Sc. Thesis, Vrije Universiteit, Brussel.

De Langhe J, Delvosalle L, Duvigneaud J, Lambinon J, Vanden Berghen C (1983). Flora Van België, Het Groothertogdom Luxemburg, Noord-Frankrijk en de Aangrenzende Gebieden, Nationale Plantentuin: Meise.

De Simone M, Morgante M, Lucchin M, Parrini P, Marocco A (1997). A first linkage map of Cichorium intybus L. using a one-way pseudo-testcross and PCR-derived markers. Mol Breed 3: 87-103.

Doyle JJ, Doyle JL (1990). Isolation of plant DNA from fresh tissue. Focus 12: 13-15

Grattapaglia D, Sederoff R (1994). Genetic linkage maps of Eucalyptus grandis and Eucalyptus urophylla using a pseudotestcross: mapping strategies and RAPD markers. Genetics 137: 1121-1137.

Grivet L, D'Hont HA, Roques D, Feldmann P, Lanaud C, Glaszmann JC (1996). RFLP mapping in cultivated sugarcane (Saccharum spp.): Genome organization in highly polyploid and aneuploid interspecific hybrid. Genetics 142: 987-2000.

Hackett CA, Bradshaw JE, Meyer RC, McNicol JW, Milbourne D, Waugh R (1998). Linkage analysis in tetraploid species: a simulation study. Genet Res Comb 71: 144-154.

Jacobs JME, Van Eck HJ, Arens P, Verker-Bakker B, Hekkert BL, Bastiaanssen HJM et al (1995). A genetic map of potato (Solanum tuberosum) integrating molecular markers, including transposons and classical markers. Theor Appl Genet 91 : 289-300.

Kosambi DD (1944). The estimation of map distances from recombination values. Ann Eugen 12: 172-175.

Lewis WH (1980). Polyploidy in angiosperms. Dicotyledons. In: Lewis WH (ed) Polyploidy, Biological Relevance, Basic Life Science, NY, Vol 13, pp 241-268.

Masterson J (1994). Stomatal size in fossil plants - evidence for polyploidy in majority of Angiosperms. Science 264: 421-423.

Mather K (1957). The Measurement of Linkage in Heredity, Methuen \& Co. Ltd, London.

Meikle RD (1992). British willows: some hybrids and some problems. Proc R Soc Edinburg Ser B 98: 13-20.

Morgante M, Vogel J (1994). Compound microsatellite primers for the detection of genetic polymorphisms. US Patent Application No. $08 / 326456$.

Neumann A (1981). Die mitteleuropäischen Salix-Arten. Mitt Forstlichen Bundes-versuchsanstalt Wien 134: 1-152 (in German).

Newsholme C (1992). Willows. The Genus Salix, Timber Press: Portland, OR.

Paiero P, Martini F (1988). I salici d'Italia, Edizioni LINT: Trieste, Italy (in Italian).

Porceddu A, Albertini E, Barcaccia G, Falcinelli M (2002). Linkage mapping in apomictic and sexual Kentucky bluegrass (Poa pratensis L.) genotypes using a two-way pseudotestcross strategy based on AFLP and SAMPL markers. Theor Appl Genet 104: 273-280.

Powell W, Machray GC, Provan J (1996). Polymorphism revealed by simple sequence repeats. Trends in Plant Sci 1: 215-222.

Qu L, Hancock JF (2001). Detecting and mapping repulsionphase linkage in polyploids with polysomic inheritance. Theor Appl Genet 103: 136-143.

Rajapakse S, Byrne DH, Zhang L, Anderson N, Arumuganathan K, Ballard RE (2001). Two genetic linkage maps of tetraploid roses. Theor Appl Genet, 103: 575-583.

Rechinger KH (1964). Salicaceae. In: Tutin, TG, Heywood, VH Burges, NA, Valentine, DH, Walters, SM, Webb, DA (eds) Flora Europea, Cambridge University Press: Cambridge, vol. 1 , pp. 43-55. 
Ripol MI, Churchill GA, Da Silva JA, Sorrells M (1999) Statistical aspects of genetic mapping in autopolyploids. Gene 235: 31-41.

Skvortsov AK (1973). Problems of Biogeocoenology, Geobotany and Plant Geography, Russian Academy of Science, Hayka Publishers: Leningrad (in Russian).

Soltis PM, Soltis DE (2000). The role of genetic and genomic attributes in the success of polyploids. Proc Natl Acad Sci USA 57: 7051-7057.

Sorrells ME (1992). Development and application of RFLPs in polyploids. Crop Sci 32, 1086-1091.

Stebbins GL (1950). Variation and Evolution in Plants, Oxford University Press: Oxford.

Thibault J (1997). Nuclear DNA amount in pure species and hybrid willows (Salix): a flow cytometric investigation. Can J Bot 76: 157-165.

Triest L, De Greef B, De Bondt R, Vanden Bossche D, D'Haeseleer M, Van Slycken J et al (1997). Use of RAPD markers to estimate hybridization in Salix alba and Salix fragilis. Belg J Bot 129: 140-148.

Triest L, De Greef B, D'Haeseleer M, Echchgadda G, Van Slycken J, Coart E (1998). Variation and inheritance of isozyme loci in controlled crosses of Salix alba and Salix fragilis. Silvae Genetica 47: 88-94.

Triest L, De Greef B, Vermeersch S, Van Slycken J, Coart E (1999). Are Salix alba and Salix fragilis a hybrid species complex? Evidence from allozyme data. Plant Syst Evol 215: 169-187.
Triest L, De Greef B, De Bondt R, Van Slycken J (2000). RAPD of controlled crosses and clones from the field suggests that hybrids are rare in the Salix alba - Salix fragilis complex. Heredity 84: 555-563.

Triest L (2001). Hybridization in staminate and pistillate Salix alba and S. fragilis (Salicaceae): morphology versus RAPD. Plant Syst Evol 226: 143-154.

Van Oijen JW, Voorrips RE (2001). JoinMap ${ }^{R}$ Version 3.0 software for the calculation of genetic linkage map. Plant Research International, Wageningen, The Netherlands.

Wu KK, Burnquist W, Sorrells ME, Tew TL, Moore PH, Tanksley SD (1992). The detection and estimation of linkage in polyploids using single-dose restriction fragments. Theor Appl Genet 83: 294-300.

Wu R, Gallo-Meagher M, Littell RC, Zeng ZB (2001). A general polyploid model for analyzing gene segregation in outcrossing tetraploid species. Genetics 159: 869-882.

Xu WW, Sleper DA, Chao S (1995). Genome mapping of polyploid tall fescue (Festuca arundinacea Schreb.) with RFLP markers. Theor Appl Genet 91: 947-955.

Yu KF, Pauls KP (1993). Segregation of random amplified polymorphic DNA markers and strategies for mapping in tetraploid alfalfa. Genome 36: 844-851.

Zabeau M, Vos P (1992). European Patent Application. Publication no. $0543858 \mathrm{Al}$. 